\title{
Transport properties of graphene functionalized with molecular switches
}

\author{
Niels Bode, ${ }^{1}$ Eros Mariani, ${ }^{2}$ and Felix von Oppen ${ }^{1}$ \\ ${ }^{1}$ Dahlem Center for Complex Quantum Systems and Fachbereich Physik, \\ Freie Universität Berlin, 14195 Berlin, Germany \\ ${ }^{2}$ Centre for Graphene Science, School of Physics, \\ University of Exeter, Stocker Rd., EX4 $4 Q L$ Exeter, UK
}

(Dated: March 20, 2012)

\begin{abstract}
We provide a theory of the electronic transport properties of a graphene layer functionalized with molecular switches. Our considerations are motivated by the spiropyran-merocyanine system which is non-polar in its ring-closed spiropyran form and zwitterionic in its ring-open merocyanine form. The reversible switching between these two isomers affects the carriers in graphene through the associated change in the molecular dipole moment, turning the graphene layer into a sensor of the molecular switching state. We present results for both the quasiclassical (Boltzmann) and the quantum-coherent regimes of transport. Quite generally, we find a linear sensitivity of the conductance on the molecular dipole moment whenever quantum interference effects play an essential role which contrasts with a quadratic (and typically weaker) dependence when quantum interference is absent.
\end{abstract}

\section{INTRODUCTION}

In recent years molecular switches on surfaces have attracted much interest due to both intrinsic scientific motivations and possible future applications in nanoelectronics. An interesting example for photochromic molecular switches are spiropyrans which allow for the reversible switching between two conformational states, a ring-closed and an open form. The chemical transformation between these two conformations can be driven thermally or by irradiation with light. In the ring-opening reaction a $\mathrm{C}-\mathrm{O}$ bond breaks, cf. Fig. 1, and the two isomers differ significantly in their geometry and electronic configurations. In particular, due to its zwitterionic form the resulting merocyanine has a large dipole moment ${ }^{1}$ which may open new design possibilities for technological applications.

In order to incorporate these switches into nanoscale devices it is of major importance to study how the molecular switches interact with a substrate. Central issues are both how the substrate affects the molecular switch and how the switch functionalizes the substrate. For instance, the substrate can modify the switching process, e.g., by steric hindrance or by quenching of excitations. ${ }^{2,3}$ Conversely, the switch may cause a reversible modification of the substrate. As an example, the optical absorption of carbon nanotubes functionalized with spiropyrans has

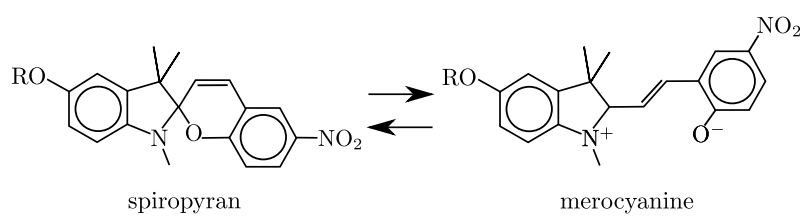

Figure 1: Switching between the ring-closed (threedimensional) spiropyran and the ring-open (planar), zwitterionic merocyanine. recently been observed to depend on the switching state. ${ }^{4}$

In this paper, we consider the electronic transport properties of graphene layers functionalized by spiropyran. Specifically, we consider how the conductance of the graphene layer differs between the switching states which are characterized by very different electric dipole moments. For isolated molecules, it is estimated ${ }^{4}$ that the dipole moment is of order of $6.2 \mathrm{D}$ in the ring-closed spiropyran form, while it is $13.9 \mathrm{D}$ in the zwitterionic merocyanine. Graphene ${ }^{5}$ provides a particularly attractive substrate for decoration with molecular switches due to its unique conduction properties, such as the absence of backscattering and an easily tunable carrier concentration, ${ }^{7,8}$ as well as its strictly two-dimensional nature. Especially this last fact suggests that the conduction properties of graphene could serve as a sensitive detector for the conformational state of the molecular switches.

Our calculations of the conductance of the graphene layer consider both the Boltzmann regime and the mesoscopic regime of coherent quantum transport. When the electronic mean free path is much larger than the Fermi wavelength and the system is sufficiently large compared to the phase coherence length, electronic transport is characterized by the quasiclassical Boltzmann conductivity. In contrast, in the mesoscopic regime, the sample size is no longer large compared to the phase coherence length, so that quantum interference effects become important and the conductance becomes sensitive to the particular impurity configuration. The average magnitude of these fluctuations about their mean value is universal and of order of the conductance quantum $e^{2} / h,^{9-12}$ and rather small changes in the precise configuration of the impurities cause significant changes in the conductance due to interference of multiple scattering trajectories. ${ }^{13,14}$ Besides the dependence on the conformational state of the decorating molecules, we also consider the influence of the densities of charge carriers and impurities, as well as the density and orientation of the 
dipolar switches.

This paper is organized as follows. We give a brief introduction to the transport properties of graphene in Sec. II. The influence of the dipoles on the conductivity is studied in Sec. III. Coherent processes are considered in Sec. IV. Some technical details of the calculations are relegated to an appendix.

\section{MODEL}

\section{A. Electronic properties of graphene}

The honeycomb lattice of graphene is formed by two inequivalent sublattices between which electrons hop as described in a tight-binding approximation. The relevant low-energy bandstructure is captured by a Dirac Hamiltonian, describing the two inequivalent Dirac cones inside the first Brillouin zone. The Hamiltonian for a single Dirac cone is given by ${ }^{5}$

$$
H_{0}=\hbar v \boldsymbol{\sigma} \cdot \boldsymbol{k},
$$

where $\boldsymbol{k}$ is the momentum, $v$ denotes the Fermi velocity and $\sigma_{\alpha}(\alpha=x, y)$ are Pauli matrices acting in the space of the two sublattices. The corresponding eigenfunctions are

$$
\boldsymbol{\psi}_{s, \boldsymbol{k}}(\boldsymbol{r})=\langle\boldsymbol{r} \mid \boldsymbol{k}\rangle=\frac{1}{\sqrt{2 \Omega}} \exp (\mathrm{i} \boldsymbol{k} \cdot \boldsymbol{r})\left(\begin{array}{c}
s \\
\mathrm{e}^{\mathrm{i} \varphi(\boldsymbol{k})}
\end{array}\right),
$$

with the linear dispersion $\epsilon_{s, \boldsymbol{k}}=\operatorname{s\hbar vk}(k=|\boldsymbol{k}|)$, where $s= \pm 1$ labels the conduction and valence bands, respectively, $\cos \varphi(\boldsymbol{k})=k_{x} / k$, and $\Omega$ is the sample area. While in principle, the relevant low energy band structure of graphene consists of two Dirac cones (valleys), we consider them to be completely decoupled as is the case in the absence of short-range scatterers. Consequently, we account for the two valleys simply through the appropriate degeneracy factor.

The concentration of charge carriers in graphene can be tuned via external gate voltages which allows one to vary the Fermi wavenumber $k_{\mathrm{F}}$. The density $n$ of conduction electrons is related to $k_{\mathrm{F}}$ through

$$
n=g \int_{0}^{\epsilon_{\mathrm{F}}} \mathrm{d} \epsilon \nu(\epsilon)=\frac{k_{\mathrm{F}}^{2}}{\pi},
$$

where $\nu(\epsilon)=\epsilon / 2 \pi(\hbar v)^{2}$ is the density of states per spin and valley, $\epsilon_{\mathrm{F}}$ is the Fermi energy, and $g=4$ accounts for the spin and valley degeneracy. We refer to Refs. 5 and 6 for a detailed review of the electronic properties of graphene.

\section{B. Green's functions}

Our discussion of the transport properties is based on a Green's function approach. The free retarded (advanced) Green's function for electrons (focusing on one
Dirac cone) is

$$
G_{0}^{R(A)}(\epsilon, \boldsymbol{k})=\frac{1}{2} \sum_{s= \pm 1} \frac{\mathbf{1}+s \boldsymbol{\sigma} \cdot \boldsymbol{k} / k}{\epsilon-\epsilon_{s, \boldsymbol{k}} \pm \mathrm{i} \eta} .
$$

The numerators in these expressions act as projectors onto states in the conduction $(s=+1)$ and valence $(s=-1)$ band, respectively. In the following we consider only electron-doped systems with a sufficiently high Fermi energy, such that all relevant processes occur in the conduction band. This allows us to restrict attention to $s=+1$ only. In fact, both our quasiclassical and our diagrammatic approaches are valid only when the system is sufficiently far from the Dirac point (characterized by electron and hole puddles in real samples ${ }^{6}$ ).

Scattering on impurities broadens the electronic spectral function, so that the impurity averaged matrix elements of the retarded (advanced) electronic Green's function become

$$
\overline{\left\langle\boldsymbol{k}^{\prime}\left|G_{\epsilon}^{R(A)}\right| \boldsymbol{k}\right\rangle}=\frac{\delta_{\boldsymbol{k}, \boldsymbol{k}^{\prime}}}{\epsilon-\epsilon_{\boldsymbol{k}} \pm \mathrm{i} \hbar / 2 \tau\left(\epsilon_{\boldsymbol{k}}\right)} \equiv \delta_{\boldsymbol{k}, \boldsymbol{k}^{\prime}} \bar{G}_{\epsilon}^{R / A}(\boldsymbol{k}) .
$$

Here, the elastic scattering time, evaluated in the Born approximation, is

$$
\frac{1}{\tau\left(\epsilon_{\boldsymbol{k}}\right)}=\frac{2 \pi}{\hbar} \sum_{\boldsymbol{k}^{\prime}} \overline{\left|\left\langle\boldsymbol{k}\left|V_{\mathrm{i}}\right| \boldsymbol{k}^{\prime}\right\rangle\right|^{2}} \delta\left(\epsilon_{\boldsymbol{k}}-\epsilon_{\boldsymbol{k}^{\prime}}\right),
$$

in accordance with Fermi's golden rule. The electronic mean free path is related to the scattering time through $l=v \tau$. The impurity potential $V_{\mathrm{i}}(\boldsymbol{r})=\sum_{j=1}^{N_{\mathrm{i}}} V(\boldsymbol{r}-$ $\left.\boldsymbol{r}_{j}\right)$ is a sum over the individual potentials $V$ of the $N_{\mathrm{i}}$ (non-magnetic) impurities which we take to be randomly distributed. Averaging over the impurity configurations (indicated by the overbar) yields

$$
\overline{\left|\left\langle\boldsymbol{k}\left|V_{\mathrm{i}}\right| \boldsymbol{k}^{\prime}\right\rangle\right|^{2}}=\frac{n_{\mathrm{i}}}{\Omega}\left|V_{\boldsymbol{k}-\boldsymbol{k}^{\prime}}\right|^{2}(1+\cos \vartheta) / 2,
$$

with $\vartheta$ the angle between $\boldsymbol{k}$ and $\boldsymbol{k}^{\prime}, V_{\boldsymbol{k}-\boldsymbol{k}^{\prime}}$ the Fourier transform of $V(\mathbf{r})$, and $n_{\mathrm{i}}=N_{\mathrm{i}} / \Omega$ the impurity density. The factor $(1+\cos \vartheta)$ reflects the absence of backscattering in graphene, even for isotropic scattering potentials.

\section{Quasiclassical transport properties}

Before discussing the influence of molecular switches on the conductivity, we briefly review the conductivity of doped graphene within a Boltzmann approach. ${ }^{7,8,15-19}$

For zero temperature, the longitudinal conductivity is given by the Kubo formula

$$
\sigma=g \frac{\hbar}{\pi \Omega} \operatorname{Tr}\left[\hat{j}_{x} \operatorname{Im} G_{\epsilon_{\mathrm{F}}}^{R} \hat{j}_{x} \operatorname{Im} G_{\epsilon_{\mathrm{F}}}^{R}\right],
$$

where the Green's functions are evaluated at the Fermi energy. The current operator is $\hat{j}_{x}=(-\mathrm{e}) \frac{\mathrm{i}}{\hbar}\left[H_{0}, x\right]=$ $(-\mathrm{e}) v \sigma_{x}$, with matrix elements

$$
\left(j_{x}\right)_{\boldsymbol{k}, \boldsymbol{k}^{\prime}}=(-\mathrm{e}) v\left\langle\boldsymbol{k}^{\prime}\left|\sigma_{x}\right| \boldsymbol{k}\right\rangle=(-\mathrm{e}) v \cos \vartheta .
$$




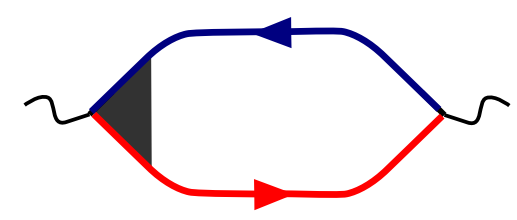

Figure 2: (Color online) Diagram for the conductivity. The conductivity loop consists of a retarded (blue) and an advanced (red) impurity averaged Green's function meeting at two current vertices. The bare current vertices are denotes by wiggly lines and the dressed vertex is defined in Fig. 3.

To lowest order, the diagram for the impurity-averaged conductivity is depicted in Fig. 2, and following standard procedures ${ }^{20}$ we obtain

$$
\bar{\sigma}=g e^{2} \nu_{0} D,
$$

with $D=v^{2} \tau_{\text {tr }} / 2$ the diffusion constant. The transport scattering rate,

$$
\begin{aligned}
\frac{1}{\tau_{\text {tr }}\left(\epsilon_{\boldsymbol{k}}\right)} & =\frac{2 \pi}{\hbar} \sum_{\boldsymbol{k}^{\prime}} \overline{\left|\left\langle\boldsymbol{k}\left|V_{\mathrm{i}}\right| \boldsymbol{k}^{\prime}\right\rangle\right|^{2}}(1-\cos \vartheta) \delta\left(\epsilon_{\boldsymbol{k}}-\epsilon_{\boldsymbol{k}^{\prime}}\right) \\
& =\frac{\pi}{\hbar} n_{\mathrm{i}} \nu\left(\epsilon_{\boldsymbol{k}}\right)\left\langle\left|V_{\boldsymbol{k}-\boldsymbol{k}^{\prime}}\right|^{2} \sin ^{2} \vartheta\right\rangle_{\vartheta},
\end{aligned}
$$

is the rate at which the memory of the $\boldsymbol{k}$-direction of the incoming electron is lost. Here, $\langle\ldots\rangle_{\vartheta}=\int_{0}^{2 \pi} \mathrm{d} \vartheta(\ldots) /(2 \pi)$ denotes an angular average over the Fermi circle. From now on, $\tau$ and $\tau_{\text {tr }}$ without explicit momentum labels are calculated at the Fermi energy. We also use the notation $\nu_{0} \equiv \nu\left(\epsilon_{\mathrm{F}}\right)$. We close this section with a brief discussion of the transport scattering time for two common sources of scattering, namely short-range and Coulomb scatterers.

\section{Short-range scatterers}

Scatterers with a short-range potential, e.g., point defects or neutral impurities, have a Fourier transform which is (approximately) independent of momentum, $V_{\boldsymbol{k}-\boldsymbol{k}^{\prime}}=V$. Thus, due to the density-of-states factor, Eq. (11) yields a transport scattering rate which is proportional to $k$ and the conductivity becomes independent of the electron density. (We assume that the potential still varies smoothly enough that the two Dirac cones remain uncoupled.)

\section{Coulomb scatterers}

Another frequent source of scattering in graphene samples are charged impurities, located at a distance $z$ above the graphene sheet. The Fourier transform of the corresponding single-impurity potential is

$$
V_{\boldsymbol{q}}^{\mathrm{c}}=\frac{2 \pi \alpha \hbar v}{q} \mathrm{e}^{-z q}
$$

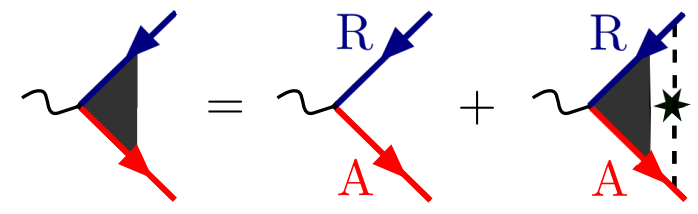

Figure 3: (Color online) Vertex corrections. The star denotes impurity scattering. Note that one only has to include diagrams with a retarded and an advanced Green's function meeting at a current vertex, see Refs. 12,21,22.

where $q=\left|\boldsymbol{k}-\boldsymbol{k}^{\prime}\right|=2 k \sin (\vartheta / 2)$ for elastic scattering processes. Here, $\alpha=e^{2} /(\hbar v \kappa)$ denotes the effective fine structure constant which involves the average dielectric constants of the neighboring media, $\kappa=\left(\kappa_{1}+\kappa_{2}\right) / 2{ }^{6,18}$ Charged impurities are screened by the conduction electrons, resulting in the effective potential $\tilde{V}_{\boldsymbol{q}}^{\mathrm{c}}=V_{\boldsymbol{q}}^{\mathrm{c}} / \varepsilon(\boldsymbol{q})$, where the dielectric function, in the limit of zero temperature, can be approximated by

$$
\varepsilon(\boldsymbol{q}) \simeq 1+q_{\mathrm{TF}} / q .
$$

This Thomas-Fermi approximation involves the characteristic wave vector $q_{\mathrm{TF}}=2 \pi \alpha \hbar v g \nu_{0} \cdot{ }^{15,16}$

The average conductivity is found to be

$$
\bar{\sigma}^{\mathrm{c}}=\frac{g e^{2}}{h} \frac{1}{\pi \alpha^{2} I_{0}} \frac{n}{n_{\mathrm{i}}^{\mathrm{c}}},
$$

which is valid at zero temperature, showing that the conductivity increases linearly with the density of charge carriers $n$ participating in the transport. This result follows from inserting the transport scattering time $\tau_{\text {tr }}^{\mathrm{c}}$ into Eq. (10). The scattering rates evaluated at the Fermi level, see Eqs. (11) and (6), are

$$
\left.\begin{array}{l}
1 / \tau_{\text {tr }}^{\mathrm{c}} \\
1 / \tau^{\mathrm{c}}
\end{array}\right\}=\frac{n_{\mathrm{i}}^{\mathrm{c}} \pi^{2} \alpha^{2} v}{2 k_{\mathrm{F}}}\left\{\begin{array}{c}
I_{0} \\
J_{0}
\end{array},\right.
$$

with $n_{\mathrm{i}}^{\mathrm{c}}$ the density of charged impurities and the abbreviations

$$
\left.\begin{array}{l}
I_{m} \\
J_{m}
\end{array}\right\}=\int_{0}^{2 \pi} \frac{\mathrm{d} \vartheta}{2 \pi} \frac{\sin ^{m}(\vartheta / 2) \mathrm{e}^{-4 z k_{\mathrm{F}} \sin (\vartheta / 2)}}{[\sin (\vartheta / 2)+g \alpha / 2]^{2}}\left\{\begin{array}{l}
\sin ^{2} \vartheta \\
1+\cos \vartheta
\end{array} .\right.
$$

For $q_{\mathrm{TF}} /\left(2 k_{\mathrm{F}}\right)=g \alpha / 2 \approx 2$ (corresponding to graphene on a $\mathrm{SiO}_{2}$ substrate $\left.^{18}\right)$ and $4 z k_{\mathrm{F}} \ll 1$ we have $I_{0} \approx 0.071$, $I_{1} \approx 0.046, I_{2} \approx 0.033, J_{0} \approx 0.18, J_{1} \approx 0.065$, and $J_{2} \approx 0.035$.

In summary, the conductivity of graphene is independent of the electron density $n$ for short-range scatterers and linear in the density for Coulomb scatterers. ${ }^{15,16,18}$ Combining both contributions, one obtains a linear rise of the conductivity which saturates at higher $n$. At low temperatures this behavior is in agreement with many experiments. ${ }^{7,8,23}$ 


\section{EFFECT OF SWITCHES ON THE CONDUCTIVITY - BOLTZMANN THEORY}

We now consider graphene samples with a dilute and random covering by molecular switches whose switching states are characterized by different electric dipole moments. ${ }^{24}$ In this section, we will assume that the electronic scattering is adequately described within a Boltzmann approach which treats consecutive scattering events as independent. We will also assume that the switching is only effected externally, e.g., by irradiation of the sample, and that all molecules are switched so that we need only discuss the conductivity for the static dipole moments associated with the two different conformations.

Consider a molecule with a nonzero electric dipole moment $\boldsymbol{d}$ attached to graphene. We assume that the electric dipole is located at a distance $z$ above the graphene sheet with the dipolar potential

$$
V^{\mathrm{d}}(\boldsymbol{r}, z)=(-e) \frac{\boldsymbol{d}_{\|} \cdot \boldsymbol{r}+d_{\perp} z}{\left(r^{2}+z^{2}\right)^{3 / 2}} .
$$

Here, $\boldsymbol{d}_{\|}$and $d_{\perp}$ are the components of the dipole moment parallel and perpendicular to the substrate, respectively, and $\boldsymbol{r}$ is a two-dimensional vector in the surface. The two dimensional Fourier transform of the dipolar potential follows readily from Gauss's law $\nabla^{2} V^{\mathrm{d}}=4 \pi e \rho$, where $\rho$ is the charge density of the dipoles. (Note that $V^{\text {d }}$ is defined as the potential energy of an electron in the field of the dipole.) Fourier transforming Gauss's law and integrating over the out-of-plane component of the wave vector, one obtains

$$
V_{\boldsymbol{q}}^{\mathrm{d}}=4 \pi \mathrm{i} e \int \frac{\mathrm{d} q_{\perp}}{2 \pi} \frac{\boldsymbol{d}_{\|} \cdot \boldsymbol{q}+d_{\perp} q_{\perp}}{q^{2}+q_{\perp}^{2}} \mathrm{e}^{\mathrm{i} q_{\perp} z},
$$

and therefore

$$
\begin{aligned}
V_{\boldsymbol{q}}^{\mathrm{d}} & =V_{\boldsymbol{q}}^{\mathrm{d}_{\|}}+V_{\boldsymbol{q}}^{\mathrm{d}_{\perp},} \\
V_{\boldsymbol{q}}^{\mathrm{d}_{\|}} & =2 \pi \mathrm{i} \alpha \hbar v\left(d_{\|} / e\right) \cos \phi \mathrm{e}^{-q z}, \\
V_{\boldsymbol{q}}^{\mathrm{d}_{\perp}} & =-2 \pi \alpha \hbar v\left(d_{\perp} / e\right) \mathrm{e}^{-q z},
\end{aligned}
$$

where $\phi$ denotes the angle between $\boldsymbol{q}$ and $\boldsymbol{d}_{\|}$. Note that $V_{\boldsymbol{q}}^{\mathrm{d} \perp}$ is real, while $V_{\boldsymbol{q}}^{\mathrm{d} \|}$ is imaginary, reflecting their symmetry properties. The resulting screened potential is given by $\tilde{V}_{\boldsymbol{q}}^{\mathrm{d}}=V_{\boldsymbol{q}}^{\mathrm{d}} / \varepsilon(q)$, with the dielectric function in Eq. (13).

We first consider a clean graphene sample where the scattering is entirely due to the decorating molecular switches with dipolar impurity potential. In such a system, the electrons are scattered at impurities with a dipole moment (but without monopole potential), and the averaged matrix elements of the impurity potential read

$$
\overline{\left|\left\langle\boldsymbol{k}\left|V_{\mathrm{i}}^{\mathrm{d}}\right| \boldsymbol{k}^{\prime}\right\rangle\right|^{2}}=\frac{n_{\mathrm{i}}^{\mathrm{d}}}{\Omega} \frac{(\alpha \hbar v \tilde{d} / e)^{2}}{1+q_{\mathrm{TF}} / q} \frac{1+\cos \vartheta}{2},
$$

with $n_{\mathrm{i}}^{\mathrm{d}}$ the density of dipoles. We use $\tilde{d}^{2}=$ $d^{2}\left(1+\cos ^{2} \xi\right) / 2$, where the angle $\xi$ measures the orientation of the dipole moment with respect to the plane such that $d_{\perp}=d \cos \xi$. To be specific, we assume that $\boldsymbol{d}_{\|}$is oriented along an arbitrary direction within the graphene layer while the perpendicular component is (approximately) the same for all dipolar switches. Inserting Eq. (22) into Eqs. (11) and (6) yields the scattering rates

$$
\left.\begin{array}{l}
1 / \tau_{\mathrm{tr}}^{\mathrm{d}} \\
1 / \tau^{\mathrm{d}}
\end{array}\right\}=2(\pi \alpha)^{2} n_{\mathrm{i}}^{\mathrm{d}} v k_{\mathrm{F}}(\tilde{d} / \mathrm{e})^{2}\left\{\begin{array}{l}
I_{2} \\
J_{2}
\end{array},\right.
$$

where $I_{2}$ and $J_{2}$ are given by Eq. (16). In the absence of other types of scatterers, Eq. (10) yields the conductivity

$$
\bar{\sigma}^{\mathrm{d}}=\frac{g e^{2}}{h} \frac{1}{(2 \pi \alpha)^{2} I_{2}} \frac{1}{n_{\mathrm{i}}^{\mathrm{d}}(\tilde{d} / e)^{2}} .
$$

Note that this result for the conductivity is independent of the electron concentration.

If the graphene sample is disordered even in the absence of the molecular switches, it is natural to consider a situation in which the dominant source of scattering is due to $N_{\mathrm{i}}^{\mathrm{c}}$ charged impurities, supplemented by $N_{\mathrm{i}}^{\mathrm{d}}$ additional dipolar scatterers. For the moment, we assume that these latter scatterers are not associated with a monopolar potential. If the distributions of these two different types of scatterers are statistically independent, the total transport scattering rate is obtained by Matthiessen's rule through adding the two individual scattering rates, $1 / \tau_{\mathrm{tr}}^{\mathrm{c}, \mathrm{d}}=1 / \tau_{\mathrm{tr}}^{\mathrm{c}}+1 / \tau_{\mathrm{tr}}^{\mathrm{d}}$. Hence, switching the dipole moments causes a relative change of the conductivity

$$
\frac{\delta \sigma}{\bar{\sigma}^{\mathrm{c}}} \simeq-2(\pi \alpha)^{2} \frac{J_{0} I_{2}}{I_{0}} \delta n_{\mathrm{i}} k_{\mathrm{F}} l_{\mathrm{c}}(\tilde{d} / e)^{2},
$$

reflecting the fact that the conductivity decreases when scatterers are added to the system. We have used $\bar{\sigma}^{\mathrm{d}} \ll$ $\bar{\sigma}^{\mathrm{c}}$ which holds for $k_{\mathrm{F}} d / \mathrm{e} \ll 1$. This limit is relevant for typical electron densities in graphene, $n \approx 10^{12} \mathrm{~cm}^{-2}$, and even rather large dipole moments $d \approx 10 \mathrm{D}$ (yielding $\left.k_{\mathrm{F}} d / e \approx 0.03\right)$. The prefactor in Eq. (25) is given by $2(\pi \alpha)^{2} J_{0} I_{2} / I_{0} \approx 1.7$ for impurities close to the graphene sheet, $z k_{\mathrm{F}} \ll 1$, see Eq. (16). Note that the mean free path $l_{\mathrm{c}}=v \tau_{\mathrm{c}}$ is proportional to $k_{\mathrm{F}}$, see Eq. (15). Thus, the influence of the dipoles on the conductivity is quadratic in $\left(k_{\mathrm{F}} d / \mathrm{e}\right)$, which increases linearly with the electron density $n$.

Frequently, an attached molecular switch will affect electronic transport not only through its dipole moment, but may also be associated with a monopolar scattering potential, e.g., due to some degree of charge transfer between graphene and the molecular switch. For this reason, we generalize our results to situations with $N_{\mathrm{i}}$ charged impurities (with screened potential $\tilde{V}_{\boldsymbol{q}}^{\mathrm{c}}$ ) and $\delta N_{\mathrm{i}}$ impurities with an additional dipole moment, where the latter also transfer an amount $\delta e=\zeta e$ of charge to the graphene. Then, the scattering potential for the latter 
takes the form $\tilde{V}_{\boldsymbol{q}}^{\mathrm{c}+\mathrm{d}}=\tilde{V}_{\boldsymbol{q}}^{\mathrm{c}}+\tilde{V}_{\boldsymbol{q}}^{\mathrm{d}}$. The corresponding transport scattering rate, Eq. (11), is

$$
\frac{1}{\tau_{\mathrm{tr}}^{\mathrm{c}+\mathrm{d}}}=\frac{1}{\tau_{\mathrm{tr}}^{\mathrm{c}}}+\frac{1}{\tau_{\mathrm{tr}}^{\mathrm{d}}}+\frac{1}{\tau_{\mathrm{tr}}^{\mathrm{c}, \mathrm{d}_{\perp}}}
$$

where $\tau_{\mathrm{tr}}^{\mathrm{c}}$ and $\tau_{\mathrm{tr}}^{\mathrm{d}}$ are given by Eqs. (15) and (23), and we introduce the shorthand

$$
\left.\begin{array}{l}
1 / \tau_{\mathrm{tr}}^{\mathrm{c}, \mathrm{d}_{\perp}} \\
1 / \tau^{\mathrm{c}, \mathrm{d}_{\perp}}
\end{array}\right\}=-2 \delta n_{\mathrm{i}} \pi^{2} \zeta \alpha v \frac{d_{\perp}}{e}\left\{\begin{array}{c}
I_{1} \\
J_{1}
\end{array},\right.
$$

with $\delta n_{\mathrm{i}}$ the density of switched molecules. This latter contribution involves interference between monopole and dipole scattering. Interestingly, $d_{\perp}$ enters linearly into this contribution while the in-plane component of the individual dipoles cancels out. This cancellation follows from the fact that $V_{\boldsymbol{q}}^{\mathrm{d}_{\|}}$in Eq. (20) is purely imaginary, while the Coulomb potential is real. $I_{1}$ and $J_{1}$ follow from Eq. (16) and we have used the (impurity averaged) matrix element of the total impurity potential

$$
\begin{aligned}
& \overline{\left|\left\langle\boldsymbol{k}\left|V_{\mathrm{i}}^{\mathrm{c}+\mathrm{d}}\right| \boldsymbol{k}^{\prime}\right\rangle\right|^{2}}=\frac{1+\cos \vartheta}{2 \Omega}\left[\left(n_{\mathrm{i}}+\delta n_{\mathrm{i}} \zeta^{2}\right)\left|\tilde{V}_{\boldsymbol{q}}^{\mathrm{c}}\right|^{2}\right. \\
& \left.+2 \delta n_{\mathrm{i}} \zeta \tilde{V}_{\boldsymbol{q}}^{\mathrm{c}} \tilde{V}_{\boldsymbol{q}}^{\mathrm{d}_{\perp}}+\delta n_{\mathrm{i}}\left|\tilde{V}_{\boldsymbol{q}}^{\mathrm{d}_{\perp}}\right|^{2}+\frac{1}{2} \delta n_{\mathrm{i}}\left|\tilde{V}_{\boldsymbol{q}}^{\mathrm{d}_{\|}}\right|^{2}\right] .
\end{aligned}
$$

The relative change of the conductivity due to switching of the dipole moments is then

$$
\frac{\delta \sigma}{\bar{\sigma}^{\mathrm{c}}} \simeq \delta n_{\mathrm{i}} \frac{2 J_{0}(\pi \alpha)^{2}}{I_{0}}\left[I_{1} \zeta l_{\mathrm{c}} d_{\perp} / e-I_{2} k_{\mathrm{F}} l_{\mathrm{c}}(\tilde{d} / e)^{2}\right],
$$

with $\delta \sigma=\left(\bar{\sigma}^{\mathrm{c}+\mathrm{d}}-\bar{\sigma}^{\mathrm{c}}\right)$ and again assuming that the switching induced change in the conductivity is small. The prefactors can be approximated by $2(\pi \alpha)^{2} J_{0} I_{1} / I_{0} \approx$ 2.3 and $2(\pi \alpha)^{2} J_{0} I_{2} / I_{0} \approx 1.7$ for $q_{\mathrm{TF}} /\left(2 k_{\mathrm{F}}\right)=g \alpha / 2 \approx 2$ and $4 z k_{\mathrm{F}} \ll 1$, see Eq. (16).

In the Boltzmann limit scattering events are independent and thus interference can only appear for scattering channels (such as monopole and dipole contributions) associated with the same scatterer. Nevertheless, our result (29) indicates that such interference contributions can significantly increase the sensitivity of the conductivity to changes of the molecular switching state. Indeed, while the change in conductivity due to switching is quadratic in the molecular dipole moment in the absence of interference, see Eqs. (24) and (25), interference gives rise to a contribution which is linear in $d_{\perp}$, and thus dominant in the relevant limit $k_{\mathrm{F}} d / e \ll 1$.

\section{EFFECT OF SWITCHES ON THE CONDUCTANCE - QUANTUM COHERENT TRANSPORT}

\section{A. Mesoscopic fluctuations}

In the previous section we have seen that interference between charge and dipole scattering originating from the same scatterer can be favorable for the readout of the switching state. Now we consider quantum coherent transport, implying that interference between partial waves scattered at different impurities becomes relevant such that one might again expect an enhanced sensitivity to the switching state of the molecules.

Because a macroscopic sample can be viewed as built of a number of mesoscopic phase coherent subsystems, which are quantum mechanically independent of each other, the macroscopic measurement effectively averages over these subsystems. The system becomes selfaveraging and is characterized by intensive quantities, such as the impurity-averaged electric conductivity $\bar{\sigma}$. At this level, the conductivity can be obtained from the Boltzmann equation, as we did in the previous section.

In contrast, in the mesoscopic regime (i.e., at sufficiently low temperatures and small system sizes where the phase coherence length becomes larger than the sample dimensions), interference between multiple scattering trajectories is important and a change in the microscopic impurity configuration or a continuous system parameter, such as the Fermi energy or an applied magnetic field, yields reproducible variations of the conductance. It is well known that the typical magnitude of these fluctuations about the mean value of the conductance is universal in the sense that it depends only on the sample geometry but is independent of the microscopic details of disorder. ${ }^{9-12}$ Importantly, such changes in the conductance are already effected by rather small changes in the impurity potential. ${ }^{13,14}$ This suggests that indeed, interference terms involving different scatterers may make a graphene sheet, in the regime of quantum coherent transport, a particularly accurate sensor of the switching state of the attached molecules. The general concepts of mesoscopic fluctuations have been introduced in Refs. 9-12 (see Ref. 20 for a pedagogical account). For the peculiarities of universal conductance fluctuations in graphene, we refer to Refs. 25-29.

\section{B. Diagrammatic calculation}

We consider charged impurities as the dominant source of scattering and an impurity potential $V_{\mathrm{i}}$, which is formed by $N_{\mathrm{i}}$ of these Coulomb scatterers. We are interested in the change of the conductance when the impurity potential changes, $V_{\mathrm{i}} \rightarrow V_{\mathrm{i}}^{\prime}$. To be specific, we assume that $\delta N_{\mathrm{i}}$ charged impurities acquire an additional dipole moment causing the change in the impurity potential. (We note that the derivation would follow the same lines, and leave our results unaffected, when dipolar impurities were added to a background of charged impurities.) For generality, we consider the correlation function of the conductance evaluated not only for different impurity potentials, but also at different Fermi energies $\epsilon_{\mathrm{F}}$ and $\epsilon_{\mathrm{F}}^{\prime}$.

A measure for the effect of the microscopic modifications is the conductivity-conductivity correlation func- 

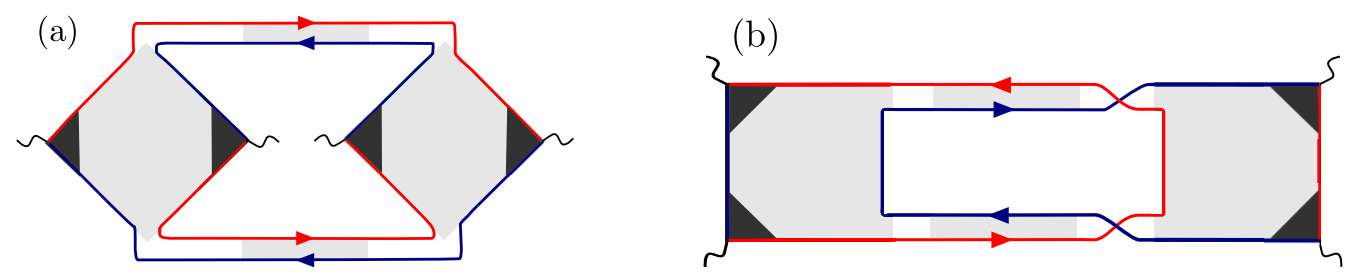

Figure 4: (Color online) Diagrams involved in the UCF. The building blocks of these diagrams are shown in Figs. 5 and 6.

tion,

$$
\overline{\Delta \sigma(\xi) \Delta \sigma\left(\xi^{\prime}\right)}=\overline{[\sigma(\xi)-\bar{\sigma}(\xi)]\left[\sigma\left(\xi^{\prime}\right)-\bar{\sigma}\left(\xi^{\prime}\right)\right]},
$$

where $\xi$ is the quantity which is modified (e.g. $\xi=$ $\left.\left\{\epsilon_{\mathrm{F}}, V_{\mathrm{i}}\right\}\right)$, and the conductivity $\sigma$ is given by Eq. (8). The diagrams representing $\overline{\Delta \sigma \Delta \sigma}$ consist of two conductivity loops (one evaluated for Fermi energy $\epsilon_{\mathrm{F}}$ and impurity potential $V_{\mathrm{i}}$, the other for $\epsilon_{\mathrm{F}}^{\prime}=\epsilon_{\mathrm{F}}+\omega$ and impurity potential $V_{i}^{\prime}$ ) which are connected by impurity lines. There are two distinct possibilities to connect the two loops, $\overline{\Delta \sigma \Delta \sigma}=\overline{\Delta \sigma \Delta \sigma}^{(a)}+\overline{\Delta \sigma \Delta \sigma}^{(b)}$, as shown in Figs. 4(a) and (b), respectively. Note that unconnected loops correspond to $\bar{\sigma}^{2}$ and hence do not enter into the variance.

Based on the standard Feynman rules for disordered systems ${ }^{20}$ Fig. 4(a) and (b) translate into the analytical expressions

$$
{\overline{\Delta \sigma(\xi) \Delta \sigma\left(\xi^{\prime}\right)}}^{(a)}=\left(\frac{g \hbar}{4 \pi \Omega}\right)^{2} 4 \beta \sum_{\boldsymbol{q}}\left(H_{1}\right)^{2}\left|\tilde{D}_{\omega}(\boldsymbol{q})\right|^{2}
$$

and

$$
{\overline{\Delta \sigma(\xi) \Delta \sigma\left(\xi^{\prime}\right)}}^{(b)}=\left(\frac{g \hbar}{4 \pi \Omega}\right)^{2} 8 \beta \sum_{\boldsymbol{q}}\left(H_{2}\right)^{2} \operatorname{Re}\left[\left(\tilde{D}_{\omega}(\boldsymbol{q})\right)^{2}\right],
$$

which are valid in the diffusive limit, $k_{\mathrm{F}} l \gg 1$ and $\omega \tau \ll 1$. The building blocks of the diagrams, the short ranged Hikami boxes $H_{1(2)}$ and the long ranged diffuson $\tilde{D}_{\omega}(\boldsymbol{q})$, are depicted in Figs. 5 and 6 . The corresponding analytical expressions are given below in Eqs. (36) and (39). The expressions for $\overline{\Delta \sigma \Delta \sigma}^{(a)}$ and $\overline{\Delta \sigma \Delta \sigma}^{(b)}$ also involve a combinatorial factor of 4 , which reflects that the diagrams in Fig. 4(a) and (b) are invariant under interchange of retarded and advanced Green functions as well as of momentum labels. In a time reversal invariant system, additional contributions stem from replacing the diffusons by Cooperons. ${ }^{20}$ This is taken care of by the symmetry factor $\beta=2$ (1) for a system with (without) time reversal invariance.

We now turn to a brief discussion of the constituents of these fluctuation diagrams, namely the diffusons $\tilde{D}_{\omega}$ and the Hikami boxes $H_{1(2)}$. The diffusons $\tilde{D}_{\omega}$ describe the diffusive motion of electrons across the sample. As depicted in Fig. 5, they are represented diagrammatically by ladder diagrams in which the two Green's functions are connected by any number of parallel impurity lines.
Analytically, this series of ladder diagrams satisfies the integral equation

$$
\begin{aligned}
& \tilde{D}_{\omega}\left(\hat{\boldsymbol{k}}, \hat{\boldsymbol{k}}^{\prime}, \boldsymbol{q}\right)=U_{2}\left(\hat{\boldsymbol{k}}-\hat{\boldsymbol{k}}^{\prime}\right) \\
& +\frac{1}{\Omega} \sum_{\hat{\boldsymbol{k}}^{\prime \prime}} \tilde{D}_{\omega}\left(\hat{\boldsymbol{k}}, \hat{\boldsymbol{k}}^{\prime \prime}, \boldsymbol{q}\right) \bar{G}_{\epsilon_{\mathrm{F}}}^{\mathrm{R}}(\boldsymbol{k}) \bar{G}_{\epsilon_{\mathrm{F}}-\omega}^{\mathrm{A}}(\boldsymbol{k}-\boldsymbol{q}) U_{2}\left(\hat{\boldsymbol{k}}^{\prime \prime}-\hat{\boldsymbol{k}}^{\prime}\right),
\end{aligned}
$$

where we leave implicit that the two Green's functions are evaluated for the impurity potentials $V_{\mathrm{i}}$ and $V_{\mathrm{i}}^{\prime}$, respectively, while impurity lines connecting them represent the correlators $\overline{V_{\mathrm{i}} V_{\mathrm{i}}^{\prime}}$. For convenience, we use the shorthand notation

$$
\begin{aligned}
& U_{1}\left(\hat{\boldsymbol{k}}-\hat{\boldsymbol{k}}^{\prime}\right)=\frac{\Omega}{2}\left(\overline{\left|\left\langle\boldsymbol{k}\left|V_{\mathrm{i}}\right| \boldsymbol{k}^{\prime}\right\rangle\right|^{2}}+\overline{\left|\left\langle\boldsymbol{k}\left|V_{\mathrm{i}}^{\prime}\right| \boldsymbol{k}^{\prime}\right\rangle\right|^{2}}\right), \\
& U_{2}\left(\hat{\boldsymbol{k}}-\hat{\boldsymbol{k}}^{\prime}\right)=\Omega \overline{\left\langle\boldsymbol{k}\left|V_{\mathrm{i}}\right| \boldsymbol{k}^{\prime}\right\rangle\left\langle\boldsymbol{k}^{\prime}\left|V_{\mathrm{i}}^{\prime}\right| \boldsymbol{k}\right\rangle} .
\end{aligned}
$$

Solving the integral equation (33), as described in App. A 1 , results in

$$
\tilde{D}_{\omega}(\boldsymbol{q}) \simeq \frac{\hbar /\left(2 \pi \nu_{0} \tau^{2}\right)}{D q^{2}-\mathrm{i} \omega+\left(\left\langle U_{1}\right\rangle /\left\langle U_{2}\right\rangle-1\right) / \tau},
$$

which is valid for small changes $\left\langle U_{1}\right\rangle-\left\langle U_{2}\right\rangle$ in the impurity configuration. Here, we have used the notation $\left\langle U_{i}\right\rangle \equiv\left\langle U_{i}\left(\hat{\boldsymbol{k}}-\hat{\boldsymbol{k}}^{\prime}\right)\right\rangle_{\hat{\boldsymbol{k}}^{\prime}}$ for the angular average, evaluated at $k=k_{\mathrm{F}}$. Explicitly evaluating these averages for our model, we obtain

$$
\left\langle U_{1}\right\rangle=\frac{\hbar}{2 \pi \nu_{0}}\left(\frac{1}{\tau^{\mathrm{c}}}+\frac{1}{2 \tau^{\mathrm{d}}}+\frac{1}{2 \tau^{\mathrm{c}, \mathrm{d}_{\perp}}}\right)
$$

and

$$
\left\langle U_{2}\right\rangle=\frac{\hbar}{2 \pi \nu_{0}}\left(\frac{1}{\tau^{\mathrm{c}}}+\frac{1}{2 \tau^{\mathrm{c}, \mathrm{d}_{\perp}}}\right) .
$$

In Eq. (36), we also introduced the scattering rate $1 / \tau=$ $(2 \pi / \hbar) \nu_{0}\left\langle U_{1}\right\rangle$.

In the fluctuation diagrams, see Fig. 4, the diffusons are connected by Hikami boxes, shown in Fig. 6, which contain the current vertices. Evaluating them in the standard manner, ${ }^{20}$ one obtains

$$
H_{1}=2 H_{2}=\frac{4 \pi}{\hbar^{3}} D \nu_{0} \tau^{2} .
$$

For the benefit of the reader, we sketch their calculation in App. A 2. 


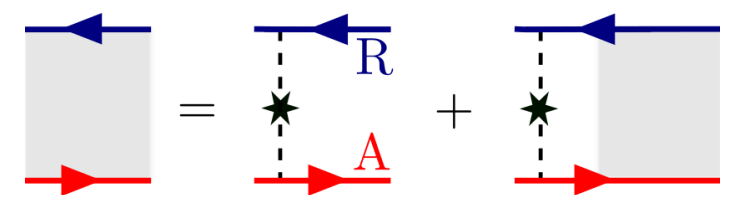

Figure 5: (Color online) Ladder series for the diffuson. The retarded (advanced) Green's function, shown in blue (red), is given by $\bar{G}_{\epsilon_{\mathrm{F}}}^{\mathrm{R}}(\boldsymbol{k})\left(\bar{G}_{\epsilon_{\mathrm{F}}-\omega}^{\mathrm{A}}(\boldsymbol{k}-\boldsymbol{q})\right)$.

We can now combine these building blocks and obtain the correlation function of the conductance. Relating conductivity and conductance through Ohm's law, $G=$ $\left(L_{y} / L_{x}\right) \sigma$, we obtain from Eqs. (31), (32), (36) and (39)

$$
\begin{aligned}
& \overline{\Delta G(\xi) \Delta G\left(\xi^{\prime}\right)}=\frac{2 \beta g^{2}}{\pi^{4}}\left(\frac{e^{2}}{h}\right)^{2} \times \\
& \times \sum_{m}\left\{\operatorname{Re}\left(\frac{1}{\lambda_{m}+\Delta \lambda}\right)^{2}+2\left|\frac{1}{\lambda_{m}+\Delta \lambda}\right|^{2}\right\} .
\end{aligned}
$$

Here, we use the abbreviations $m=\left\{m_{x}, m_{y}\right\}$ and

$$
\begin{aligned}
& \lambda_{m}=\left(m_{x}\right)^{2}+\left(m_{y} L_{x} / L_{y}\right)^{2}, \\
& \Delta \lambda=-\frac{L_{x}^{2}}{D \pi^{2}}\left[\mathrm{i} \omega+\frac{1}{\tau}\left(1-\left\langle U_{1}\right\rangle /\left\langle U_{2}\right\rangle\right)\right] .
\end{aligned}
$$

The boundary conditions of the system, namely perfect leads and hard walls at the transverse boundaries, imply the quantization $q_{\alpha}=m_{\alpha} \pi / L_{\alpha}(\alpha=x, y)$ with $m_{x}=$ $\{1, \ldots, \infty\}$ and $m_{y}=\{0,1, \ldots, \infty\}$. We note that both the Hikami boxes and the diffusons depend separately on the microscopic details of the sample, as encapsulated in the scattering rates and the density of states. Nevertheless, in the conductance-conductance correlation function, Eq. (40), these quantities cancel against each other such that all microscopic details enter only through $\Delta \lambda$.

\section{Results}

In order to see the influence of the quantum coherent processes, we first review ${ }^{20,25-29}$ the variance of the conductance, $\overline{(\Delta G)^{2}}=\overline{[G-\bar{G}]^{2}}$, describing the average magnitude of the universal conductance fluctuations. From Eq. (40) (with $\Delta \lambda=0$ ) we obtain

$$
\overline{(\Delta G)^{2}}=\frac{6 \beta}{\pi^{4}}\left(\frac{g e^{2}}{h}\right)^{2} \sum_{m} \frac{1}{\left(\lambda_{m}\right)^{2}}=\beta \eta\left(\frac{g \mathrm{e}^{2}}{h}\right)^{2},
$$

where $\eta \simeq 1 / 15$ for $L_{x} \gg L_{y}$, and $\eta \approx 0.1$ for a square device. Hence the amplitude of the fluctuations depends on the sample geometry but is universal in the sense, that it is independent of the electron concentration and the microscopic type or configuration of disorder. Comparing the amplitude of the fluctuations with the average

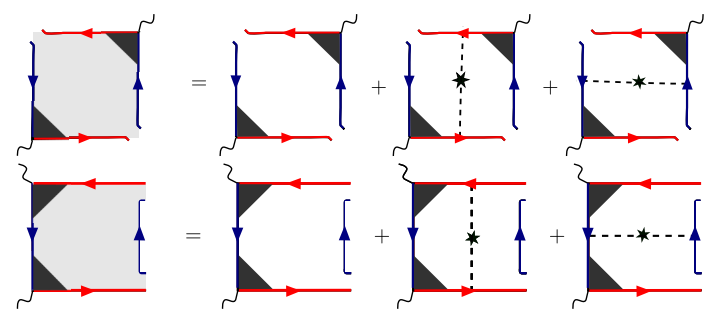

Figure 6: (Color online) Hikami boxes $H_{1}$ (top) and $H_{2}$ (bottom).

conductance yields

$$
\frac{\sqrt{\overline{(\Delta G)^{2}}}}{\bar{G}}=\frac{2 I_{0} \sqrt{\beta \eta}}{J_{0}} \frac{L_{x}}{L_{y}} \frac{1}{k_{\mathrm{F}} l_{\mathrm{c}}}
$$

again assuming that charged scatterers, cp. Eq. (14), predominantly limit the conductance. For a square device this yields $\sqrt{\overline{(\Delta G)^{2}}} / \bar{G} \approx 0.35 /\left(k_{\mathrm{F}} l_{\mathrm{c}}\right)$. This indicates that for charged impurities, the quantum coherent processes are more important at large Fermi wavelengths and high impurity concentrations (albeit such that our underlying assumption of $k_{\mathrm{F}} l_{\mathrm{c}} \gg 1$ still holds).

Switching the decorating molecules affects the correlation function $\overline{\Delta G\left(V_{\mathrm{i}}\right) \Delta G\left(V_{\mathrm{i}}^{\prime}\right)}$, see Eq. (40), via a change of the diffuson pole,

$$
\begin{aligned}
\Delta \lambda & =\frac{L_{x}^{2}}{D \pi^{2}} \frac{\left\langle U_{1}\right\rangle-\left\langle U_{2}\right\rangle}{\left\langle U_{2}\right\rangle} \frac{1}{\tau} \simeq \frac{L_{x}^{2}}{D \pi^{2}} \frac{1}{2 \tau^{\mathrm{d}}} \\
& \simeq\left(2 \alpha^{2} I_{0} J_{2} / J_{0}\right)\left(L_{x} / L_{y}\right) \delta N_{\mathrm{i}}\left(k_{\mathrm{F}} / l_{\mathrm{c}}\right)(\tilde{d} / e)^{2},
\end{aligned}
$$

see Eq. (42). Note that this depends linearly on the number of switches $\delta N_{\mathrm{i}}$ and is independent of the electron concentration. Again, we assume that the monopole contribution of the charged impurities predominantly limits the conductivity, and thus $\left(\left\langle U_{1}\right\rangle-\left\langle U_{2}\right\rangle\right) /\left\langle U_{2}\right\rangle$ is a small quantity. The prefactor can be approximated by $\left(2 \alpha^{2} I_{0} J_{2} / J_{0}\right) \approx 0.028$, see Eq. (16). The dependence of $\overline{\Delta G\left(V_{\mathrm{i}}\right) \Delta G\left(V_{\mathrm{i}}^{\prime}\right)}$ on $\Delta \lambda$ can be easily evaluated numerically and is depicted in Fig. 7. One finds that the correlation function varies linearly with $\Delta \lambda$ for small modifications of the microscopic impurity configuration. We thus conclude that the typical variation of the conductance with switching state is given by

$$
\frac{\sqrt{\left[\overline{\left[G\left(V_{\mathrm{i}}\right)-G\left(V_{\mathrm{i}}^{\prime}\right)\right]^{2}}\right.}}{\sqrt{\overline{(\Delta G)^{2}}}} \simeq \chi \frac{\sqrt{\delta N_{\mathrm{i}}}}{\sqrt{k_{\mathrm{F}} l_{\mathrm{c}}}}\left(k_{\mathrm{F}} \tilde{d} / e\right),
$$

where we lumped the numerical prefactors into $\chi=$ $2 \alpha \sqrt{\left(I_{0} J_{2} / J_{0}\right) L_{x} / L_{y}}$ which is $\chi \approx 0.23$ for a square device. Note that Eq. (46) depends linearly on the dipole moment $d$. In contrast to the Boltzmann interference result, this also holds for dipoles which are oriented parallel to the graphene sheet, $d=d_{\|}$, as well as for molecular 


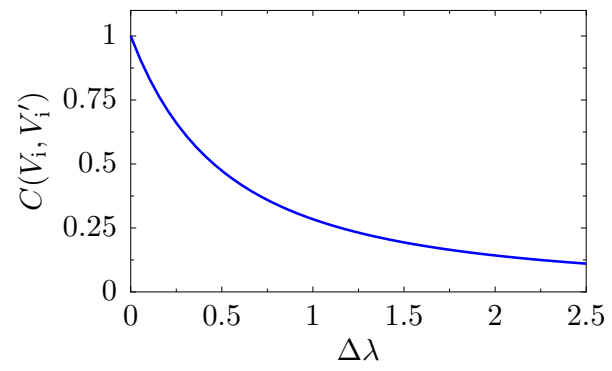

Figure 7: (Color online) The normalized correlation function $C\left(V_{\mathrm{i}}, V_{\mathrm{i}}^{\prime}\right)=\overline{\Delta G\left(V_{\mathrm{i}}\right) \Delta G\left(V_{\mathrm{i}}^{\prime}\right)} / \overline{(\Delta G)^{2}}$, plotted as a function of $\Delta \lambda$.

switches which are pure dipole scatterers. We also note that in the Boltzmann limit, the changes of the conductance scale with $\delta n_{\mathrm{i}}$ and are larger for clean devices and large electron densities, see Eq. (29). In contrast, in the mesoscopic regime larger impurity concentrations $n_{\mathrm{i}}$ and small Fermi wavelengths are favorable for the effect of interference, and the changes are proportional to $\sqrt{\delta N_{\mathrm{i}}}$, cf. Eq. (46).

\section{SUMMARY AND CONCLUSIONS}

Due to its two-dimensional nature, graphene layers are attractive substrates for functionalization by molecular switches. In this paper, we considered how the conductance of a graphene layer depends on the switching state of the decorating molecules. Specifically, we considered the spiropyran-merocyanine system where the two switching states affect the charge carriers via large changes in the electric dipole moment. We find that the sensitivity of the electronic properties of the graphene layer to the switching state is particularly strong when quantum interference is relevant in the transport process. This strong sensitivity is expressed as a linear dependence on the change in dipole moment, which contrasts with a quadratic dependence when quantum interference is ineffective.

Within a quasiclassical Boltzmann approach, quantum interference has to emerge from scattering on a single impurity. In this case, we find a strong sensitivity to the switching state when the molecular switches cause scattering of carriers both through a monopolar contribution (e.g., due to charge transfer between graphene and the molecular switches) as well as a dipolar contribution. Specifically, the interference contribution involving the corresponding scattering amplitudes is nonzero whenever the molecular dipole moment has a component perpendicular to the graphene layer.

In mesoscopic samples, interference contributions to the conductance (universal conductance fluctuations) are well known to be sensitive to small changes in the impurity potential. This provides an alternative mechanism for how the conductance is affected by the molecu- lar switching state. We find that again, these quantum interference contributions result in a switching-induced change of the conductance which is linear in the change of the molecular dipole moment, albeit with a random sign.

The quasiclassical and the mesoscopic regimes differ in the dependence of the sensitivity on other parameters such as the doping level of graphene or the coverage with molecular switches. In the Boltzmann limit high densities of electrons and switches, but otherwise clean samples, are favorable for the read-out of the switching state. Assuming a perpendicular dipole moment and charge transfer to the graphene, we estimate that a density of switches $\delta n_{\mathrm{i}} \approx 5 \times 10^{10} \mathrm{~cm}^{-2}$ is required for changing the conductivity by $1 \%$, see Eq. (29). (Here we assume $n \approx 10^{12} \mathrm{~cm}^{-2}, l \approx 50 \mathrm{~nm}$ and $d \approx 10 \mathrm{D}$.) The mesoscopic contribution becomes particularly important in samples with low carrier density. In this regime, the conductance of mesoscopic samples (here assuming $L_{x}=L_{y} \approx 5 \mu \mathrm{m}$ ) would be modified by $10 \%$ of the UCF even for moderately low densities of switches $\delta n_{\mathrm{i}} \approx 10^{9} \mathrm{~cm}^{-2}$, as indicated by Eq. (46).

\section{Acknowledgments}

We acknowledge discussions with J. P. Rabe which triggered our interest in dipolar switches on graphene and are grateful for financial support by the Deutsche Forschungsgemeinschaft through SFB 658.

\section{Appendix A: Details of the calculation}

In this appendix we sketch the derivations of the diffuson and the Hikami boxes, which are used in Sec. IV. We follow standard procedures,${ }^{20}$ which have been also applied to the study of graphene. ${ }^{25-29}$

\section{Diffuson}

The ladder series for the diffuson, Eq. (33), is depicted in Fig. 5. For low temperatures we consider only processes at the Fermi energy, yielding

$$
\int \mathrm{d} \epsilon_{k} \nu\left(\epsilon_{k}\right) \bar{G}_{\epsilon_{\mathrm{F}}}^{\mathrm{R}}(\boldsymbol{k}) \bar{G}_{\epsilon_{\mathrm{F}}-\omega}^{\mathrm{A}}(\boldsymbol{k}-\boldsymbol{q}) \simeq \frac{f_{\omega}(\hat{\boldsymbol{k}}, \boldsymbol{q})}{\left\langle U_{1}\right\rangle} .
$$

Here we use the abbreviation

$$
f_{\omega}(\hat{\boldsymbol{k}}, \boldsymbol{q})=1+\mathrm{i} \omega \tau-(v \tau)^{2}(\boldsymbol{q} \cdot \hat{\boldsymbol{k}})^{2}-\mathrm{i} v \tau \boldsymbol{q} \cdot \hat{\boldsymbol{k}},
$$

where the approximation holds in the limit of small $\omega$ and $\boldsymbol{q}$. Hence, the ladder series for the diffuson, Eq. (33), becomes

$$
\begin{aligned}
& \tilde{D}_{\omega}\left(\hat{\boldsymbol{k}}, \hat{\boldsymbol{k}}^{\prime}, \boldsymbol{q}\right)=U_{2}\left(\hat{\boldsymbol{k}}-\hat{\boldsymbol{k}}^{\prime}\right) \\
& +\frac{1}{\left\langle U_{1}\right\rangle}\left\langle\tilde{D}_{\omega}\left(\hat{\boldsymbol{k}}, \hat{\boldsymbol{k}}^{\prime \prime}, \boldsymbol{q}\right) f_{\omega}\left(\hat{\boldsymbol{k}}^{\prime \prime}, \boldsymbol{q}\right) U_{2}\left(\hat{\boldsymbol{k}}^{\prime \prime}-\hat{\boldsymbol{k}}^{\prime}\right)\right\rangle_{\hat{\boldsymbol{k}}^{\prime \prime}}
\end{aligned}
$$


cf. for example the derivation in Ref. 20. First we average over $\hat{\boldsymbol{k}}$ in order to obtain $\tilde{D}_{\omega}\left(\hat{\boldsymbol{k}}^{\prime}, \boldsymbol{q}\right) \equiv\left\langle\tilde{D}_{\omega}\left(\hat{\boldsymbol{k}}, \hat{\boldsymbol{k}}^{\prime}, \boldsymbol{q}\right)\right\rangle_{\hat{\boldsymbol{k}}}$ which satisfies the integral equation

$$
\begin{aligned}
& \tilde{D}_{\omega}\left(\hat{\boldsymbol{k}}^{\prime}, \boldsymbol{q}\right)=\left\langle U_{2}\right\rangle \\
& +\frac{1}{\left\langle U_{1}\right\rangle}\left\langle\tilde{D}_{\omega}\left(\hat{\boldsymbol{k}}^{\prime \prime}, \boldsymbol{q}\right) f_{\omega}\left(\hat{\boldsymbol{k}}^{\prime \prime}, \boldsymbol{q}\right) U_{2}\left(\hat{\boldsymbol{k}}^{\prime \prime}-\hat{\boldsymbol{k}}^{\prime}\right)\right\rangle_{\hat{\boldsymbol{k}}^{\prime \prime}}
\end{aligned}
$$

We approximate

$$
\tilde{D}_{\omega}\left(\hat{\boldsymbol{k}}^{\prime}, \boldsymbol{q}\right) \simeq \tilde{D}_{\omega}(\boldsymbol{q})+2 \hat{\boldsymbol{k}}^{\prime} \cdot\left\langle\hat{\boldsymbol{k}}^{\prime} \tilde{D}_{\omega}\left(\hat{\boldsymbol{k}}^{\prime}, \boldsymbol{q}\right)\right\rangle_{\hat{\boldsymbol{k}}^{\prime}}
$$

and introduce the shorthand $\tilde{D}_{\omega}(\boldsymbol{q}) \equiv\left\langle\tilde{D}_{\omega}\left(\hat{\boldsymbol{k}}^{\prime}, \boldsymbol{q}\right)\right\rangle_{\hat{\boldsymbol{k}}^{\prime}}$. Then, averaging over $\hat{\boldsymbol{k}}^{\prime}$ results in

$$
\begin{aligned}
\tilde{D}_{\omega}(\boldsymbol{q})= & \left\langle U_{2}\right\rangle+\tilde{D}_{\omega}(\boldsymbol{q}) \frac{\left\langle U_{2}\right\rangle}{\left\langle U_{1}\right\rangle}\left(1+\mathrm{i} \omega \tau-\frac{(v \tau q)^{2}}{2}\right) \\
& -\mathrm{i} v \tau \boldsymbol{q} \cdot\left\langle\hat{\boldsymbol{k}}^{\prime} \tilde{D}_{\omega}\left(\hat{\boldsymbol{k}}^{\prime}, \boldsymbol{q}\right)\right\rangle_{\hat{\boldsymbol{k}}^{\prime}}
\end{aligned}
$$

Multiplying Eq. (A5) with $\hat{\boldsymbol{k}}^{\prime}$ and then averaging over $\hat{\boldsymbol{k}}^{\prime}$ yields

$$
\left\langle\hat{\boldsymbol{k}}^{\prime} \tilde{D}_{\omega}\left(\hat{\boldsymbol{k}}^{\prime}, \boldsymbol{q}\right)\right\rangle_{\hat{\boldsymbol{k}}^{\prime}}=\gamma\left(\left\langle\hat{\boldsymbol{k}}^{\prime} \tilde{D}_{\omega}\left(\hat{\boldsymbol{k}}^{\prime}, \boldsymbol{q}\right)\right\rangle_{\hat{\boldsymbol{k}}^{\prime}}-\mathrm{i} \boldsymbol{q}(v \tau / 2) \tilde{D}_{\omega}(\boldsymbol{q})\right),
$$

with $\gamma=\left\langle\hat{\boldsymbol{k}} \cdot \hat{\boldsymbol{k}}^{\prime} U_{2}\left(\hat{\boldsymbol{k}}-\hat{\boldsymbol{k}}^{\prime}\right)\right\rangle_{\hat{\boldsymbol{k}}^{\prime}} /\left\langle U_{1}\right\rangle$. Multiplication of the last line with $\boldsymbol{q}$ yields

$$
\boldsymbol{q} \cdot\left\langle\hat{\boldsymbol{k}}^{\prime} \tilde{D}_{\omega}\left(\hat{\boldsymbol{k}}^{\prime}, \boldsymbol{q}\right)\right\rangle_{\hat{\boldsymbol{k}}^{\prime}}=-\mathrm{i} \frac{v \tau q^{2}}{2} \frac{\gamma}{1-\gamma} \tilde{D}_{\omega}(\boldsymbol{q})
$$

Plugging this result into Eq. (A6) brings after straightforward algebra Eq. (36) for the diffuson $\tilde{D}_{\omega}(\boldsymbol{q})$, as stated in the main text.

\section{Hikami boxes}

We evaluate the Hikami boxes, labeled $H_{1}$ and $H_{2}$, respectively, which are depicted in Fig. 6. Approximating $k_{\mathrm{F}} l \gg 1$ and $\omega \tau \ll 1$, three diagrams contribute to leading order to the Hikami boxes $H_{i}=H_{i}^{(a)}+H_{i}^{(b)}+H_{i}^{(c)}$, with $i=1,2$.
The diagram $H_{2}^{(a)}$ consists of two retarded and two advanced Green's functions with momentum $\boldsymbol{k}$. In contrast to the evaluation of the diffuson, the Hikami boxes are rather short-ranged and we can neglect the $\boldsymbol{q}$ dependences. Because of the vertex corrections a factor $\tau_{\text {tr }} / \tau$ comes with each of the current vertices so that we obtain

$$
\begin{aligned}
H_{2}^{(a)} & =\left(e v \frac{\tau_{\mathrm{tr}}}{\tau}\right)^{2} \sum_{\boldsymbol{k}} \cos ^{2} \vartheta\left(\bar{G}_{\epsilon_{\mathrm{F}}}^{R}(\boldsymbol{k}) \bar{G}_{\epsilon_{\mathrm{F}}}^{A}(\boldsymbol{k})\right)^{2} \\
& =2 \pi\left(e v \frac{\tau_{\mathrm{tr}}}{\tau}\right)^{2} \nu_{0} \tau^{3}
\end{aligned}
$$

assuming $\left(U_{1}-U_{2}\right)$ to be small.

The diagram $H_{2}^{(b)}$ consists of twice two retarded and one advanced Green's function with the same wave vector, respectively, and an additional impurity cross, so that

$$
\begin{aligned}
& H_{2}^{(b)}=\left(e v \frac{\tau_{\mathrm{tr}}}{\tau}\right)^{2} \sum_{\boldsymbol{k}} \cos ^{2} \vartheta\left(\bar{G}_{\epsilon_{\mathrm{F}}}^{R}(\boldsymbol{k})\right)^{2} \bar{G}_{\epsilon_{\mathrm{F}}}^{A}(\boldsymbol{k}) \times \\
& \times \sum_{\boldsymbol{k}^{\prime}} n_{\mathrm{i}}\left|V_{\boldsymbol{q}}\right|^{2} \frac{1+\cos \vartheta^{\prime}}{2}\left(\bar{G}_{\epsilon_{\mathrm{F}}}^{R}\left(\boldsymbol{k}^{\prime}\right)\right)^{2} \bar{G}_{\epsilon_{\mathrm{F}}}^{A}\left(\boldsymbol{k}^{\prime}\right) \\
& \quad=-\frac{1}{2} H_{2}^{(a)} .
\end{aligned}
$$

Here we have replaced the sum by an integral and used that the integration over two retarded and one advanced Green's function yields a factor $-\mathrm{i}(\tau)^{2}$ while the extra factor $\left|V_{\boldsymbol{q}}\right|^{2}$ contributes a factor $1 / \tau$.

The calculation of $H_{2}^{(c)}$ follows similar lines, but here the two current vertices are evaluated at different wave vectors with the consequence that the extra $\left|V_{\boldsymbol{q}}\right|^{2}$ yields a factor $\left(1 / \tau-1 / \tau_{\text {tr }}\right)$, resulting in

$$
H_{2}^{(c)}=\left(\frac{\tau_{\mathrm{tr}}}{\tau}-1\right) H_{2}^{(a)}
$$

In a similar manner the other type of Hikami boxes, $H_{1}$, is evaluated. One finds $H_{1}^{(a)}=H_{2}^{(a)}$, and $H_{1}^{(b)}=$ $H_{1}^{(c)}=H_{2}^{(c)}$. Combining these contributions yields Eq. (39) of the main text for the Hikami boxes.
1 J. Whelan, D. Abdallah, J. Wojtyk, and E. Buncel, J. Mater. Chem. 20, 5727 (2010).

2 M. Piantek, G. Schulze, M. Koch, K. J. Franke, F. Leyssner, A. Krüger, C. Navio, J. Miguel, M. Bernien, M. Wolf, W. Kuch, P. Tegeder, and J. I. Pascual, J. Am. Chem. Soc. 131, 12729 (2009).

3 C. Bronner, G. Schulze, K. J. Franke, J. I. Pascual, and P. Tegeder, J. Phys.: Condens. Matter 23, 484005 (2011).

${ }^{4}$ E. Malic, C. Weber, M. Richter, V. Atalla, T. Klamroth, P. Saalfrank, S. Reich, and A. Knorr, Phys. Rev. Lett. 106, 097401 (2011).
5 A. H. C. Neto, F. Guinea, N. M. R. Peres, K. S. Novoselov, and A. K. Geim, Rev. Mod. Phys. 81, 109 (2009).

${ }^{6}$ S. Das Sarma, S. Adam, E.H. Hwang and E. Rossi, Rev. Mod. Phys. 83, 407 (2011).

7 K. S. Novoselov, A. K. Geim, S. V. Morozov, D. Jiang, Y. Zhang, S. V. Dubonos, I. V. Grigorieva, and A. A. Firsov, Science 306, 666 (2004).

${ }^{8}$ K. S. Novoselov, A. K. Geim, S. V. Morozov, D. Jiang, M. I. Katsnelson, I. V. Grigorieva, S. V. Dubonos, and A. A. Firsov, Nature 438, 197 (2005).

9 P. A. Lee and A. D. Stone, Phys. Rev. Lett. 55, 1622 
(1985).

10 B. Altshuler, JETP Lett. 41, 648 (1985).

11 P. A. Lee, A. D. Stone, and H. Fukuyama, Phys. Rev. B 35, 1039 (1987).

12 C. L. Kane, R. A. Serota, and P. A. Lee, Phys. Rev. B 37, 6701 (1988).

13 B. L. Al'tshuler and B. Z. Spivak, JETP Lett. 42, 447 (1985).

14 S. Feng, P. A. Lee, and A. D. Stone, Phys. Rev. Lett. 56, 1960 (1986).

15 T. Ando, J. Phys. Soc. Jap. 75, 074716 (2006).

${ }^{16}$ K. Nomura and A. H. MacDonald, Phys. Rev. Lett. 96, 256602 (2006).

17 E. McCann, K. Kechedzhi, V.I. Fal'ko, H. Suzuura, T. Ando, and B.L. Altshuler, Phys. Rev. Lett. 97, 146805 (2006).

18 S. Adam, E. H. Hwang, V. Galitski, and S. Das Sarma, PNAS 104, 18392 (2007).

19 M. Auslender and M. I. Katsnelson, Phys. Rev. B 76, 235425 (2007).

20 E. Akkermans and G. Montambaux, Mesoscopic Physics of Electrons and Photons (Cambridge University Press, 2007).
21 B. L. Al'tshuler and B. I. Shklovskii, JETP 64, 127 (1986).

${ }^{22}$ H. U. Baranger and A. D. Stone, Phys. Rev. B 40, 8169 (1989).

23 J. H. Chen, C. Jang, M. S. Fuhrer, E. D. Williams, and M. Ishigami, Nature Phys. 4, 377 (2008).

${ }^{24}$ In the spiropyran-merocyanine system, the switching states differ most significantly in their corresponding dipole moments and thus higher order terms in a multipole expansion of the charge distribution can presumably be neglected.

25 K. Kechedzhi, O. Kashuba, V.I. Fal'ko, Phys. Rev. B 77, 193403 (2008).

${ }^{26}$ K. Kechedzhi, D. W. Horsell, F. V. Tikhonenko, A. K. Savchenko, R. V. Gorbachev, I. V. Lerner, and V. I. Fal'ko, Phys. Rev. Lett. 102, 066801 (2009).

27 M. Y. Kharitonov and K. B. Efetov, Phys. Rev. B 78, 033404 (2008).

28 A. Rycerz, J. Tworzydlo, and C. W. Beenakker, Europhys. Lett. 79, 57003 (2007).

29 J. Berezovsky, M.F. Borunda, E.J. Heller, and R.M. Westervelt, Nanotechnology 21, 274013 (2010). 